\title{
Hematemesis from ruptured aberrant right hepatic artery aneurysm eroding through the duodenal wall
}

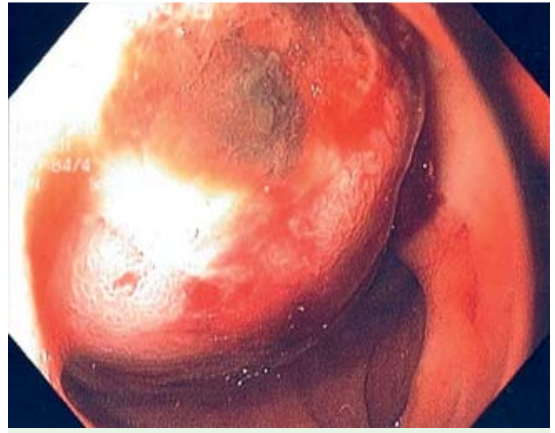

Fig. 1 Large submucosal mass with ulcer in the duodenal bulb.

A 56-year-old man presented with hematemesis and multiple episodes of melena. He had a history of chronic lymphocytic leukemia and traumatic rupture of the spleen leading to splenectomy and splenic artery embolization 6 years earlier.

Esophagogastroduodenoscopy (EGD) revealed a large submucosal mass $(7 \times 5 \mathrm{~cm})$ with an ulcerated overlying area associated with clot in the duodenal bulb ( $\bullet$ Fig. 1). The ulcer was treated with epinephrine $(1: 10000)$. A computed tomography (CT) scan of the abdomen revealed a $2.5 \times 1.8-\mathrm{cm}$ pseudoaneurysm from an aberrant hepatic artery off the superior mesenteric artery ( $\bullet$ Fig. 2 ) along with surrounding hematoma, causing mass effect on the duodenum; this was further confirmed with a CT angio$\operatorname{gram}($ Fig. 3 a).

Coil embolization was performed with complete obliteration of the hepatic artery pseudoaneurysm ( $\bullet$ Fig. $\mathbf{3 b}$ ). The patient was subsequently discharged home after 4 days of observation.

The patient presented 2 months later with recurrent episodes of melena. A CT angiogram showed no active extravasations. EGD revealed a long segment of coil protruding from the pylorus into the stomach, along with coffee ground materials. There was a large mound-like focal bulge at the superior aspect of the duodenal bulb, with a 6-mm defect without active bleeding, along with the protruding coil ( $\bullet$ Fig. 4). The patient underwent a distal gastrectomy, Billroth II gastrojejunos-

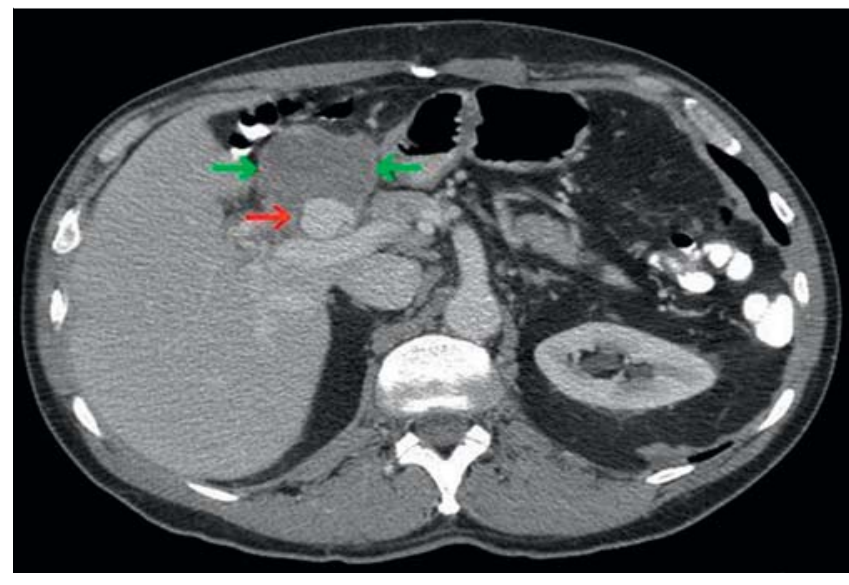

Fig. 2 An oral and intravenous contrastenhanced computed tomography (CT) scan demonstrates a pseudoaneurysm (red arrow) with a surrounding hematoma (green arrows) just above the duodenal bulb and medial to the left lobe of the liver.
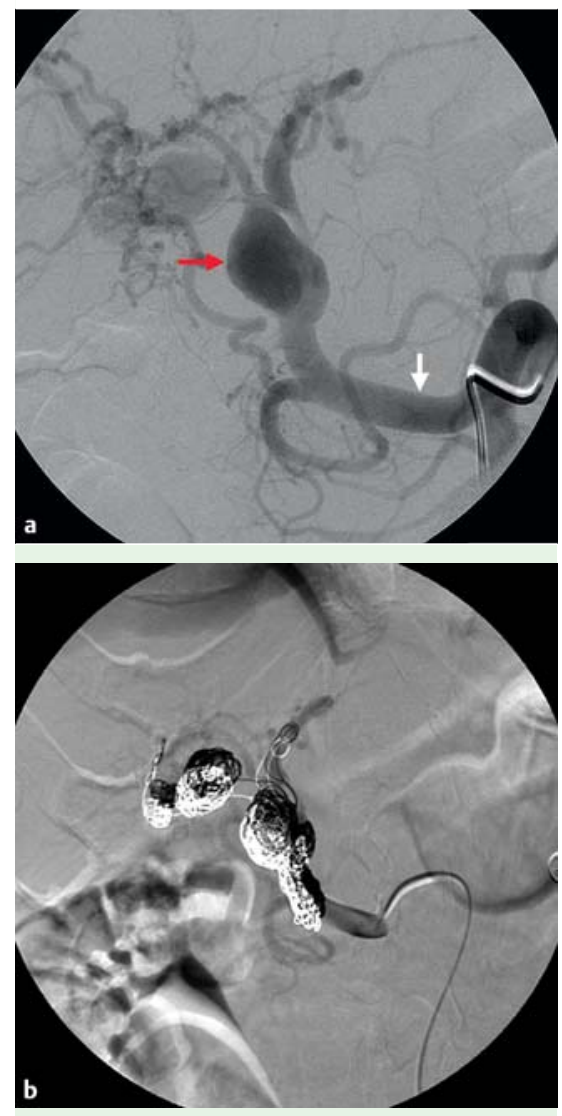

Fig. 3 a An angiogram performed immediately after the computed tomography (CT) scan confirms the pseudoaneurysm (red arrow) arising from a replaced hepatic artery (white arrow) off the superior mesenteric artery. b Post-embolization arteriogram of the common hepatic artery, demonstrating occlusion of the two large pseudoaneurysms.

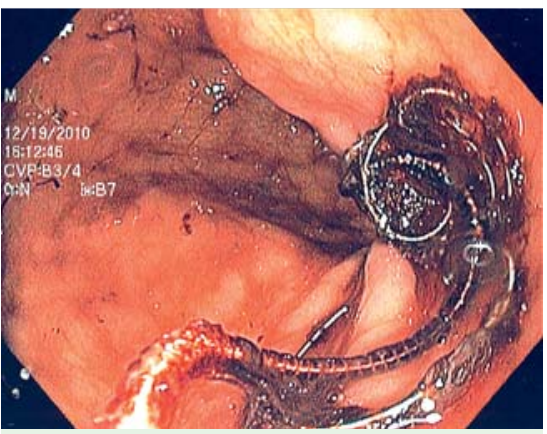

Fig. 4 Long segment of coil protruding from the pylorus into the stomach.

tomy, and ligation of gastroduodenal artery. He was discharged after 5 days of observation and remained well without further episodes of bleeding after 6 months of follow-up.

Hepatic artery pseudoaneurysm is a rare cause of upper gastrointestinal bleeding, and can be life-threatening [1,2]. Angiographic embolization is an effective method of treatment with a reported success rate of $80-100 \%$ [3]. However, complications from embolization are not unusual, as noted in our case with extrusion of coils through the duodenal wall with potential for re-bleeding. Surgery may be needed in unusual circumstances for more definitive therapy.

Endoscopy_UCTN_Code_CCL_1AB_2AZ_3AD

Competing interests: None 
Y. J. Kim¹, S. K. Satapathy², L. Law ${ }^{2}$, A. Volfson ${ }^{2}$, B. Friedman ${ }^{3}$, S. Yang ${ }^{2}$, C. Sung ${ }^{3}$, D. S. Siegel ${ }^{3}$, B. DeVito ${ }^{4}$

1 Department of Internal Medicine, Hofstra North Shore-LIJ Health system at Long Island Jewish Medical Center, New York, USA

2 Division of Gastroenterology, Hofstra North Shore-LIJ Health system at Long Island Jewish Medical Center, New York, USA

3 Department of Radiology, Hofstra North Shore-LIJ Health system at Long Island Jewish Medical Center, New York, USA

4 Division of Gastroenterology, Hofstra North Shore-LIJ Health system at North Shore University Hospital, New York, USA

\section{References}

1 Poon $R$, Tuen H, Yeung $C$ et al. GI haemorrhage from fistula between right hepatic artery pseudoaneurysm and the duodenum secondary to acute cholecystitis. Gastrointest Endosc 2000; 51: 491 - 493

2 Lumsden AB, Mattar SG, Allen RC, Bacha EA. Hepatic artery aneurysms: the management of 22 patients. J Surg Res 1996; 60: 345-350

3 Nicholson T, Travis S, Ettles D et al. Hepatic artery angiography and embolization for hemobilia following laparoscopic cholecystectomy. Cardiovasc Intervent Radiol 1999; 22: $20-24$

\section{Bibliography}

DOI $10.1055 / \mathrm{s}-0030-1256736$

Endoscopy 2011; 43: E323 - E324

(c) Georg Thieme Verlag KG Stuttgart · New York . ISSN 0013-726X

\section{Corresponding author} S. K. Satapathy, MD

Division of Gastroenterology Hofstra North Shore-LIJ Health system at Long Island Jewish Medical Center 270-05 76th Avenue

New Hyde Park

New York 11040

USA

Fax: +1-718-343-0128

sanjaya.satapathy@yahoo.com 